\section{MOUNT WILSON OBSERVATORY}

The positions and areas of sun spots furnished by the Mount Wilson Observatory for publication in the Monthly Weather Review are taken from the daily records of the magnetic polarities of sun spots. These records are made at the 150 -foot telescope by projecting the image of the sun, mean diameter 42.8 centimeters, upon a sheet of paper and sketching the spots. The heliographic latitude and longitude of each group is read from a disk on which the circles of solar longitude and the parallels of latitude have been drawn for every 10 degrees. Twenty of these disks are available for different diameters of the image and different values of the heliographic latitude of the sun's center.

To determine the areas a réseau, ruled to square millimeters, is placed on the sketch and the area of each group counted in square millimeters. The areas are then reduced to millionths of the visible hemisphere, taking into account the actual diameter of the image. The distance of each group from the center of the disk is then measured and the projection factors obtained to correct the areas for foreshortening.

The solar photographs made at the 60-foot tower telescope, sun's mean diameter 17.1 centimeters, are compared with the sketch and, if any spots have been omitted, they are measured on the photograph by methods similar to those described in the Greenwich Photoheliographic Results.-Seth B. Nicholson.

Positions and areas of sun spots

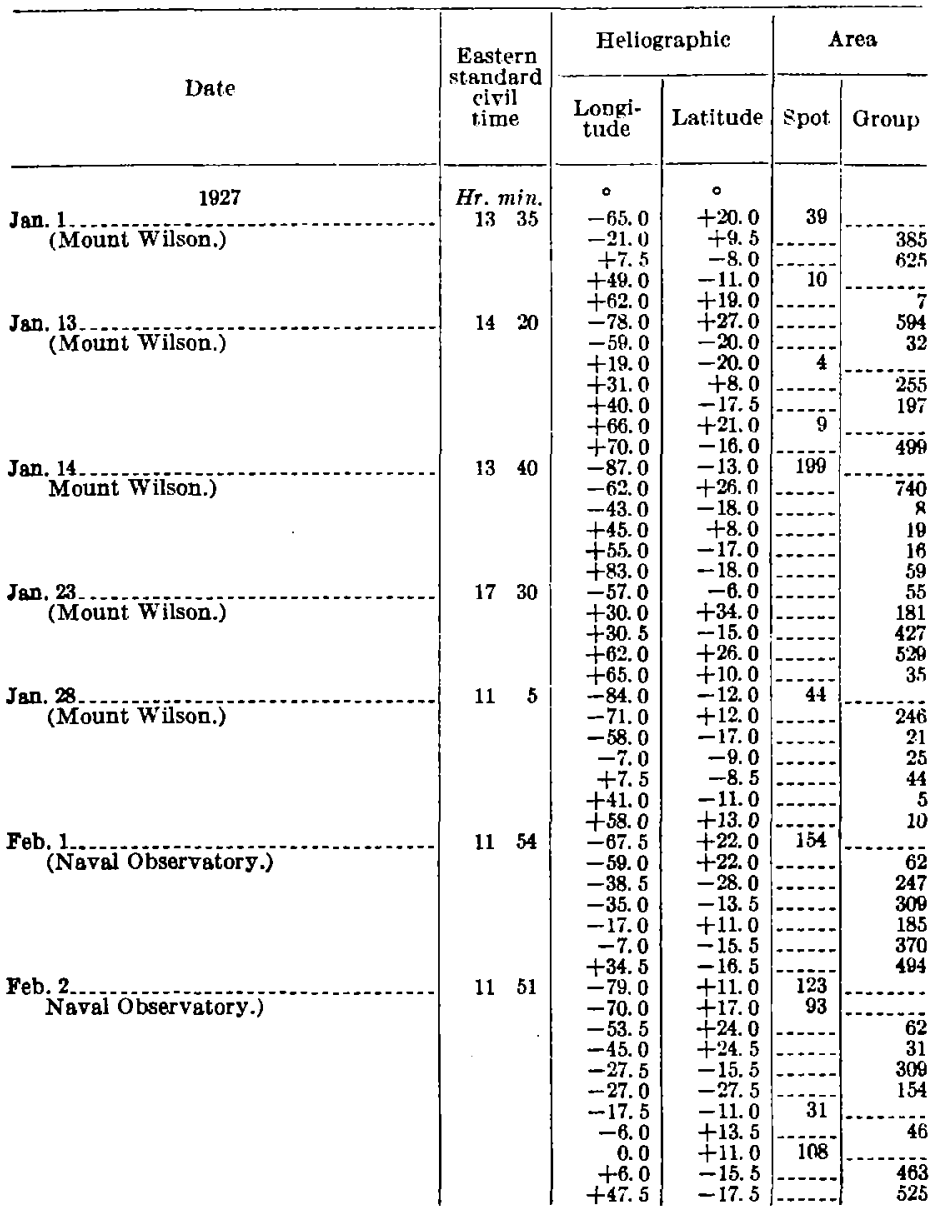

Positions and areas of sun spots-Continued

\begin{tabular}{|c|c|c|c|c|c|c|}
\hline \multirow{2}{*}{ Date } & \multirow{2}{*}{\multicolumn{2}{|c|}{$\begin{array}{c}\text { Eastern } \\
\text { standard } \\
\text { civil } \\
\text { time }\end{array}$}} & \multicolumn{2}{|c|}{ Heliographic } & \multicolumn{2}{|c|}{ Area } \\
\hline & & & $\begin{array}{l}\text { Longi- } \\
\text { tude }\end{array}$ & Latitude & Spot & Group \\
\hline & \multirow{2}{*}{\multicolumn{2}{|c|}{$\begin{array}{l}H r . \min \\
11 \\
47\end{array}$}} & 0 & $\circ$ & & \\
\hline \multirow{8}{*}{ Feb. (Naval Observatory.) } & & & -80.0 & $\begin{array}{r}-15.0 \\
0.5\end{array}$ & 154 & \\
\hline & & & -57.5 & $\begin{array}{r}+15.0 \\
+15.0\end{array}$ & 154 & - \\
\hline & & & $\begin{array}{l}-40.5 \\
-32.0\end{array}$ & $\begin{array}{l}+22.5 \\
+24.5\end{array}$ & $\begin{array}{c}15 \\
\ldots . . .\end{array}$ & 26 \\
\hline & & & $\begin{array}{l}-18.0 \\
-14.0\end{array}$ & $\begin{array}{l}+23.0 \\
-16.0\end{array}$ & $\cdots . .$. & $\begin{array}{r}62 \\
\mathbf{3 0 0}\end{array}$ \\
\hline & & & $\begin{array}{r}-14.0 \\
-4.5\end{array}$ & -28.0 & & 123 \\
\hline & & & $\begin{array}{r}+8.0 \\
\end{array}$ & +15.5 & 10 & nes \\
\hline & & & $\begin{array}{r}+12.5 \\
+19.5\end{array}$ & $\begin{array}{l}+11.0 \\
-160\end{array}$ & & \\
\hline & & & +61.0 & -17.5 & $\cdots$ & \\
\hline \multirow{6}{*}{ Feb. 4 (Naval Óbservatory.) } & 135 & & $\begin{array}{l}-70.0 \\
-49.5\end{array}$ & $\begin{array}{l}-14.0 \\
+10.0\end{array}$ & 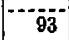 & \\
\hline & & & $\begin{array}{l}-42.5 \\
-17.0\end{array}$ & $\begin{array}{r}+15.5 \\
+25.0\end{array}$ & 31 & 83 \\
\hline & & & -5.5 & +24.0 & 15 & 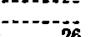 \\
\hline & & & +1.0 & $\begin{array}{r}-10.0 \\
-10.0\end{array}$ & -..- & $\begin{array}{r}260 \\
370\end{array}$ \\
\hline & & & $\begin{array}{r}+10.0 \\
+10.5\end{array}$ & -11.0 & $\cdots$ & $\begin{array}{l}62 \\
15\end{array}$ \\
\hline & & & $\begin{array}{r}+3.0 \\
+35.5\end{array}$ & $\begin{array}{l}+15.0 \\
-15.0\end{array}$ & $\begin{array}{c}80 \\
-\cdots\end{array}$ & $-27 \overline{8}$ \\
\hline \multirow{4}{*}{$\begin{array}{l}\text { Feb. } 5 .(\text { Harvard.) } \\
\text { (a) }\end{array}$} & & 21 & $\begin{array}{l}+75.0 \\
-51 .\end{array}$ & $\begin{array}{l}-18.0 \\
-13 .\end{array}$ & $\cdots$ & $\begin{array}{l}463 \\
362\end{array}$ \\
\hline & & & $\begin{array}{l}-34 . \\
-28 .\end{array}$ & $\begin{array}{l}+10 . \\
+15\end{array}$ & 73 & \\
\hline & & & $\begin{array}{l}+12 . \\
+15\end{array}$ & -14 & - & 438 \\
\hline & & & +38 & $\begin{array}{r}+8 . \\
+9 .\end{array}$ & 174 & $\cdots$ \\
\hline \multirow{7}{*}{ Feb. (Mount Wilson.) } & 182 & 20 & -67.0 & +13.0 & 10 & 188 \\
\hline & & & $\begin{array}{l}-4.0 \\
-40.0\end{array}$ & $\begin{array}{r}-8.0 \\
-14.0\end{array}$ & $\cdots$ & $\begin{array}{r}10 \\
781\end{array}$ \\
\hline & & & $\begin{array}{l}-24.5 \\
-2.0\end{array}$ & $\begin{array}{r}\mathbf{+ 7 . 5} \\
-12.5\end{array}$ & $\cdots$ & $\begin{array}{l}14 \\
26\end{array}$ \\
\hline & & & -20.0 & $\begin{array}{l}+10.0 \\
+18.0\end{array}$ & 83 & . \\
\hline & & & +12.0 & $\begin{array}{l}+10.0 \\
+26.0\end{array}$ & $\ldots . .$. & 271 \\
\hline & & & $\begin{array}{r}+30.0 \\
+33.0\end{array}$ & $\begin{array}{l}-17.0 \\
-28.0\end{array}$ & .......... & 618 \\
\hline & & & +58.0 & +12.0 & 133 & $\ldots$ \\
\hline \multirow{7}{*}{ Feb. 7 (Naval Observatory.) } & 11 & 53 & -50.0 & $\begin{array}{r}-10.0 \\
+14.0\end{array}$ & at & \\
\hline & & & $\begin{array}{l}-33.0 \\
-30.5\end{array}$ & $\begin{array}{r}-7.5 \\
-14.0\end{array}$ & $\cdots$ & $\begin{array}{r}62 \\
556 \\
88\end{array}$ \\
\hline & & & $\begin{array}{l}-15.5 \\
-11.0\end{array}$ & $\begin{array}{r}-12.5 \\
+8.0\end{array}$ & 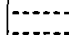 & $\begin{array}{c}62 \\
108 \\
\end{array}$ \\
\hline & & & -1.5 & $\begin{array}{r}+16.0 \\
\end{array}$ & $\cdots$ & 300 \\
\hline & & & $\begin{array}{r}+38.0 \\
+38.0\end{array}$ & ${ }_{-17.5}^{+250}$ & ......... & $\begin{array}{r}46 \\
370\end{array}$ \\
\hline & & & $\begin{array}{r}+43.0 \\
+66.0\end{array}$ & $\begin{array}{r}-28.0 \\
+11.0\end{array}$ & 123 & 46 \\
\hline & & & +78.5 & $=-13.0$ & 175 & 154 \\
\hline \multirow{6}{*}{ Feb. 8 (Harvard.) } & 10 & $\mathbf{3 1}$ & -53. & +20 & $\ldots$. & 52 \\
\hline & & & -17 & -6. & 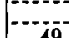 & $\begin{array}{r}80 i \\
72\end{array}$ \\
\hline & & & +3 & +6 & ${ }^{30}$ & 76 \\
\hline & & & $\begin{array}{r}+4 \\
+12 .\end{array}$ & $\begin{array}{r}+8 \\
+14 .\end{array}$ & 46 & 201 \\
\hline & & & $\begin{array}{l}+40 \\
+47\end{array}$ & -18. & 362 & \\
\hline & & & $\begin{array}{l}+51 \\
+78\end{array}$ & -30. & 88 & $\cdots$ \\
\hline \multirow{6}{*}{ Feb. 9 (Naval öbservatory.) } & 114 & 48 & -67.0 & $\begin{array}{l}+27.5 \\
-8.0\end{array}$ & 123 & $\cdots$ \\
\hline & & & $\begin{array}{l}-4.5 \\
-4.0\end{array}$ & $\begin{array}{r}-8.0 \\
-15.0\end{array}$ & $\mid-\ldots .$. & $\begin{array}{r}93 \\
463\end{array}$ \\
\hline & & & $\begin{array}{r}+16.0 \\
+18.5\end{array}$ & $\begin{array}{r}+5.5 \\
+10.0\end{array}$ & $4 \overline{6}$ & 278 \\
\hline & & & +26.5 & +17.0 & $|\ldots . .|$. & 216 \\
\hline & & & $\begin{array}{r}0.00 \\
+58.0\end{array}$ & +25.5 & 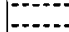 & 123 \\
\hline & & & $\begin{array}{l}+0 . \\
+70\end{array}$ & -28.0 & 43 & 309 \\
\hline \multirow{5}{*}{ Feb. ${ }_{\text {(Naval Observatory.) }}$} & 11 & 48 & -54.5 & -28.0 & 123 & \\
\hline & & & $\begin{array}{r}+8.5 \\
+\mathbf{8 . 5}\end{array}$ & $\begin{array}{r}-8.0 \\
-15.5\end{array}$ & $\cdots$ & $\begin{array}{r}83 \\
432\end{array}$ \\
\hline & & & $\begin{array}{r}+28.0 \\
+31.5\end{array}$ & $\begin{array}{r}+5.5 \\
+10.0\end{array}$ & 46 & Al \\
\hline & & & $\begin{array}{l}+40.0 \\
+69.0\end{array}$ & $\begin{array}{l}+17.0 \\
+250\end{array}$ & 123 & 154 \\
\hline & & & $\begin{array}{r}+82.0 \\
+82.0\end{array}$ & -17.0 & 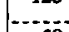 & 300 \\
\hline \multirow{5}{*}{$\begin{array}{l}\text { Feb. } 11 \\
\text { (Naval Observatory.) }\end{array}$} & & 47 & $\begin{array}{l}+83.0 \\
-83.0\end{array}$ & $\begin{array}{l}-29.0 \\
-13.0\end{array}$ & $\begin{array}{r}62 \\
123\end{array}$ & \\
\hline & & & -41.0 & -28.0 & 123 & \\
\hline & & & $\begin{array}{l}+24.0 \\
+440\end{array}$ & -15.0 & . & 370 \\
\hline & & & +45.5 & +10.0 & 62 & W \\
\hline & & & $\begin{array}{r}+5.0 . \\
+59.5\end{array}$ & $\begin{array}{l}+10.0 \\
+18.0\end{array}$ & 62 & 238 \\
\hline \multirow{4}{*}{ Feb. 12} & 11 & 56 & -68.0 & $\begin{array}{l}-13.0 \\
-0.5\end{array}$ & 123 & \\
\hline & & & $\begin{array}{r}+37.5 \\
+37.5\end{array}$ & $\begin{array}{l}-8.0 \\
-8.0\end{array}$ & $\cdots$ & 185 \\
\hline & & & $\begin{array}{r}+39.0 \\
+58.5\end{array}$ & $\begin{array}{r}-13.5 \\
++6.5\end{array}$ & $\mid \cdots$ & $\begin{array}{l}401 \\
154\end{array}$ \\
\hline & & & $\begin{array}{l}+58.0 \\
+70.0\end{array}$ & $\begin{array}{l}+10.5 \\
+17.5\end{array}$ & $\mid \begin{array}{l}46 \\
\ldots . . . .\end{array}$ & $1 \quad 128$ \\
\hline
\end{tabular}

